\title{
Variability in Quasar Broad Absorption Line Outflows
}

\author{
Daniel M. Capellupo ${ }^{1}$, Fred Hamann ${ }^{1}$, Joseph C. Shields ${ }^{2}$, \\ Tom A. Barlow ${ }^{3}$, and Paola Rodriguez ${ }^{4}$ \\ ${ }^{1}$ Department of Astronomy, University of Florida, Gainesville, FL 32611-2055, USA \\ Email: dancaps@astro.ufl.edu \\ ${ }^{2}$ Department of Physics \& Astronomy, Ohio University, Athens, OH 45701, USA \\ ${ }^{3}$ California Institute of Technology, 1200 E. California Blvd., Pasadena, CA 91125, USA \\ ${ }^{4}$ Department of Astronomy and Astrophysics, Pennsylvania State University, 525 Davey Lab, \\ University Park, PA 16802, USA
}

Keywords. galaxies: active, quasars: general, quasars: absorption lines

Broad absorption lines (BALs) in quasar spectra identify high-velocity outflows that likely exist in all quasars and could play a major role in feedback to galaxy evolution (e.g., Di Matteo et al. 2005). Studying the variability in these BALs can help us understand the structure, evolution, and basic physical properties of these outflows. We are investigating BAL variability in a sample of 25 luminous quasars at $1.2<z<2.9$ with multi-epoch observations that cover time scales from less than around a month to 7.7 years in the quasar rest-frame. We investigate changes in the C IV $\lambda 1549$ BALs, and we see a variety of phenomena, including some BALs that either appeared or disappeared completely and other BALs that did not change at all over the whole observation period. Our first results are for two subsamples of observations, one where the time baseline is 4 to 9 months (short-term) and the other where the time baseline is $\sim 4$ to 6 years (long-term). We find that $65 \%$ of the quasars varied in the long-term data, while $44 \%$ varied in the short-term data. Typically, only portions of the BAL troughs vary. We find that BALs at higher outflow velocities are more likely to vary than those at lower velocities and that weaker BALs are more likely to vary than stronger BALs. We also find that the fractional change in BAL strength correlates with the strength of the BAL feature, but not with the outflow velocity. These results are currently being prepared for publication (Capellupo et al., in preparation).

We also have some preliminary results from our study of the entire dataset. We plot the fraction of quasars with CIV BAL variability versus the time interval between observations. This fraction decreases for shorter time scales, but we still find variability in an interval as short as 26 days. This implies that we have not yet found a time scale threshold below which there is no variability. We plan to expand on this work, in particular continuing the study of the time scales of variability and also investigating variability in the Si IV BALs in order to help define the actual time scales, the causes of the variability, and the sizes, locations, and volatility of the outflowing gas.

\section{References}

Capellupo, D. M., et al. 2010, in preparation

Di Matteo, T., Springel, V., \& Hernquist, L. 2005, Nature, 433, 604 